\title{
Peter Sloterdijk: la técnica como ejercicio ontológico
}

\author{
Peter Sloterdijk: Technique \\ as Ontological Exercise
}

\author{
Leopoldo Tillería Aqueveque \\ Instituto Nacional de Capacitación Profesional | Chile \\ Contacto: leopoldo.tilleria@inacapmail.cl
}

\section{Resumen}

En este trabajo, se discute la relación entre la ontología de las esferas y el papel de la técnica en el pensamiento de Sloterdijk, considerando la idea de ejercicio como forma de equipamiento inmunológico. Para ello, se parte de la premisa sloterdijkiana de que el ser humano se constituye esencialmente a partir de la noción de espacio. La técnica, entonces, debiera entenderse en un sentido bastante más sofisticado que el de una simple transformación ingeniosa de las fuerzas de la naturaleza. Al respecto, se confronta la comprensión de la técnica de Sloterdijk con la meditación de la técnica de Ortega, en particular, con su conocida idea de "sobrenaturaleza”. Este parangón constata la importancia de la artificialidad en la deriva técnico/tecnológica del ser humano, teniendo en cuenta precisamente el concepto de domo o invernadero como correlato casi perfecto de la definición orteguiana de la técnica. Así, desde el útero materno hasta una caminata espacial, siempre habrá un otro que nos constituye, que nos define, que nos "acoraza" decisivamente. A partir de esta suerte de fenomenología acrobática, se concluye con la pregunta acerca de la posibilidad de interpretar la teoría del alemán de la mano del diseño y la estética, o mejor aún, aferrados a la erótica.

Palabras clave: esfera, espacio, inmunización, ontología, Sloterdijk, técnica

\section{Abstract}

The relationship between the ontology of the spheres and the role of technique in Sloterdijk's thought is discussed, considering the idea of exercise as a form of im- 
munological equipment. To do this, we start from the Sloterdijkian premise that the human being is essentially constituted from the notion of space. Technique, then, should be understood in a far more sophisticated sense than that of a simple ingenious transformation of the forces of nature. In this regard, the understanding of Sloterdijk's technique is confronted with the meditation of Ortega's technique, in particular, with his well-known idea of "supernature". This paragon confirms the importance of artificiality in the technical/technological drift of the human being, taking into account precisely the concept of dome or greenhouse as an almost perfect correlate of Ortega's definition of technique. Thus, from the womb to a space walk, there will always be an other that constitutes us, that defines us, that decisively "armor" us. Starting from this sort of acrobatic phenomenology, we conclude with the question about the possibility of interpreting the theory of the German thinker hand in hand of design and aesthetics, or better yet, clinging to erotic.

Keywords: sphere, space, immunization, ontology, Sloterdijk, technique

\section{Introducción}

En la conferencia del 19 de mayo de 2000, en el Centro de Estudios Europeos de la Universidad de Harvard, el filósofo alemán Peter Sloterdijk (2006a) decía en una retórica muy heideggeriana lo siguiente: "El destierro de los hábitos de apariencia humanística es el acontecimiento lógico principal de nuestro tiempo, un acontecimiento ante el que es inútil buscar refugio en argumentos de buena voluntad. Pero este destierro va más allá: alcanza a todas las ilusiones del sercabesímismo [BeisichSein]" (2). Iniciaba de esta manera una crítica a la cultura tecnológica, que culminaría con una exhortación de la tecnología postclásica y de lo que él llama artes auténticas. Poner en cuestión este compromiso técnico-natural de la posmodernidad, sería caer en la sospecha de un anacronismo que negaría las actuales posibilidades tecnológicas y que, al mismo tiempo, dejaría estancadas cuestiones menos "duras", como la política, la ética, la estética, las artes y — ¡cómo no! - la religión. La pregunta fundamental, entonces, es qué tiene que ver la técnica con este "destierro de los hábitos humanistas" de los que habla Sloterdijk, o, si se quiere ver de otra forma, cuál es el papel de lo humano en la perspectiva de la técnica del filósofo germano. Más allá de la nutrida camada de detractores que tiene en el establishment intelectual europeo, sobre todo en la izquierda alemana, Sloterdijk, como bien dice Meaney (2018), no es conocido por una sola gran tesis, sino por una explosión de metralla de acuñaciones impresionistas (antropotécnica, ginecología negativa, coinmunidad) 
que sugieren la presencia al acecho de algún sistema más grande. Es más, el que en Estados Unidos haya sido considerado el principal pensador público del país y uno de los diez intelectuales más importantes de la revista Foreign Policy, probablemente tenga que ver con una razón más pop-cultural que de otro tipo, si se tiene en cuenta que la intelectualidad anglófona solo es cautivada por una figura extranjera cuando puede aislarse de sus pensamientos e ideas algún tipo de meme o conceptobyte pegajoso, moderno y provocativo (Raschke, 2013: 1).

Lo que nos interesa es dilucidar el camino que recorre Sloterdijk como aclaración de la relación entre ser humano y mundo circundante por medio de la técnica. Ante la "monstruosidad" de la obra sloterdijkiana (Oosterling, 2007), mediática, mística, y, en sus propias palabras, considerada como una "filosofía poetizada", no nos queda más remedio que acercarnos lo más cautelosamente posible. Sírvanos de argumento la idea de Van Tuinen: "Los recursos teóricos utilizados [por Sloterdijk] son extremadamente diversos y van desde el psicoanálisis y la filosofía constructivista hasta la teología, Filosofía india, teoría arquitectónica, paleoantropología, etnología, cultura pop, medicina, economía, teoría de los medios, teoría de sistemas y la cibernética" (2007: 279). Pues bien, para asegurar una hermenéutica lo suficientemente aclaradora, nuestra lectura de Sloterdijk se hará a través de una cierta confrontación con la filosofía de Ortega, una de las meditaciones más influyentes sobre la técnica del siglo xx, y que en cierto modo comparte con los desarrollos de Sloterdijk la idea de una técnica esencialmente entretejida con la vida humana. De esta manera, el "problema técnico" quedaría justamente circunscrito al pensar contemporáneo.

Cuando la humanidad se enfrenta a la explosión de sus posibilidades técnicas (por ejemplo, desde el punto de vista biotecnológico o nanotecnológico), lo que en definitiva hace es enfrentarse a la constitución de un ser humano que para Sloterdijk nunca está acabado. Tanto así, que la pregunta por la técnica en nuestro autor, después de todo, supone que no tenemos solo la geosfera y la biosfera en sus estados originales como legado de la evolución, sino que la tecnosfera y la noosfera, en cuanto intervenciones humanas a través de la ciencia y la tecnología, fueron introducidas en el mundo y aún se desconoce hasta qué punto pueden transformar la propia geosfera y la biosfera (Tomaz, 2020: 5). Sin ir más lejos, la concepción de Sloterdijk de terrorismo deja ver la incidencia de la idea de atmósfera en las sociedades actuales: "Sólo se entiende el terrorismo cuando se le concibe como una forma de investigación del medio ambiente bajo el punto de vista de su destructibilidad" (2006d: 88). En otras palabras, se trata del principio de ataque al medioambiente y a la defensa inmunológica de un organismo, de donde el filósofo preferirá acuñar el término atmoterrorismo. 
En el trabajo se aborda, en primer lugar, la "capa” mayor de la filosofía de Sloterdijk, su teoría de las esferas, que representa, centrada en la noción de espacio como conformación originaria, una perspectiva ontológica post-cartesiana y en gran medida post-epistémica (Raschke, 2013). Enseguida, se discute el papel que juegan en la teoría antropotécnica las ideas de técnica y de sistemas inmunitarios a propósito de la noción de domo o caparazón. La última sección intenta vincular "técnicamente" los conceptos de crianza preparatoria y artificialidad.

\section{Ontología, esferología, espacio}

El proyecto filosófico de Sloterdijk supone una nueva ontología de lo humano: "En Esferas se plantea al mismo tiempo "una onto y filogénesis de los espacios de vida humana” (Safranski, 2009: 16)” (Sordo y Guzmán Díaz, 2013: 143). En palabras de Huerta Rodríguez (2016), "Es bastante significativo el análisis microsferológico de ginecología negativa que realiza en torno al ser que aún no es arrojado al mundo y cuyo medio de existencia es intrauterino, acústico y acuoso-sanguíneo; es decir, que sería un ser-todavía-no-en-el-mundo pero que ya se constituye ontológicamente como ser-en, específicamente como ser-en un espacio intrauterino" (18). En lo que a primera vista resulta una confrontación directa con Heidegger, Sloterdijk dirá que el ser humano se constituye fundamentalmente a partir de la idea de espacio. Esta es la premisa fundamental del concepto de esfera: somos seres humanos en el espacio. Las esferas son entonces espacios de florecimiento de la especie humana, ámbitos sincronizados de participación. Justamente dicha noción de esfera escapa a cualquier tipo de representación ontológica o epistemológica —incluso física - que quisiera ver en ella una suerte de cápsula espacial que "entra” en contacto con el resto de las entidades, humanas o no. Sloterdijk (2003) ha dejado bien en claro que siempre estamos envueltos extáticamente en relaciones esféricas:

La espacialidad interior animada, que intentaremos mostrar en todos los aspectos fundamentales de la existencia y de la cultura humanas, es efectivamente un realissimum que se substrae en principio a toda representación geométrica y lingüística - en general a toda representación-, y que, sin embargo, gracias a una potencia de redondeamiento que actúa antes de todas las construcciones circulares formales y técnicas, fuerza en cualquier sitio donde haya un ente algo así como conformaciones originarias de círculos y globos. (81) 
Bajo esta idea, burbujas, globos y espumas son los diferentes tipos de espacios ontológicos construidos por el ser humano, que le sirven para habitar el mundo, sobrevivir en él y desarrollar su proyecto de vida, y que deben ser interpretados en clave de sistemas inmunológicos, esto es, como espacios elaborados con el propósito de generar lugares habitables en los que poder coexistir (Gimeno Monfort y López Frías, 2018). Sloterdijk pensará el concepto de esfera no de manera territorial, sino más bien relacional: "Quizás sea incluso mejor compararlo con una red de relaciones, que de alguna manera nos preocupa como seres humanos y en que los seres humanos emergen como un nodo entre otros de la densificación de la red" (Ernste, 2018: 274). Deliberadamente o no, en su ontología de las esferas, presentada en los tres volúmenes de su portentoso Esferas, Sloterdijk sigue el modelo trinitario que tantas veces ha servido a la filosofía y la teología para describir sus objetos fundamentales (las tres críticas de Kant o la trinidad de claridad, simplicidad y funcionalidad del Tractatus, por ejemplo). En el primer volumen, Esferas I: Burbujas. Microsferología (2003), el filósofo germano sienta las bases de una ontología arcaica de la co-subjetividad. Sloterdijk sugiere la tesis de que literalmente nacemos de una burbuja (el útero materno) e incluso que somos despojados de la placenta protectora para después hacer una inmersión "artificialmente" individuada en el mundo exterior. A decir de Arango, Sloterdijk "plantea la situación históricoespacial del hombre como habitante de placentas semióticas - a más de las amnióticas. [...] Fundamenta una 'ginecología negativa' en la que la madre no será nunca mostrada como mujer física con hijos, sino como espacialidad inmunitaria" (2007: 219).

Ahora, será en Esferas II: Globos. Macrosferología (2004) donde Sloterdijk tematizará las grandes esferas, mostrando, por así decir, la extensión biopolítica de la naturaleza humana. Dichas esferas muestran cómo el ser-en a pequeña escala retorna a una relación política y cósmica, pero, en este caso, por medio de una remisión a lo grande y máximo (Sloterdijk, 2003). Se trata de la acción por medio de la cual toda vida pasa de su caverna corporal originaria a un receptáculo social. Escribe Sloterdijk (2004): "A partir de entonces [de la entrada en vigor del racionalismo filosófico europeo] hay que concebir las esferas como globos en el sentido preciso de la palabra y no ya como contornos pregeométricos, psicocosmológicos, del mundo próximo junto con sus extensiones arquitectónicas, morfológicamente poco nítidas" (338). Significa, dice Sloterdijk (2004), el tránsito de la esfera como espacio de la ética y erótica altruistas a la macrosfera como matriz de la globalización. Por último, en Esferas III. Espumas. Esferología plural Sloterdijk recurrirá al concepto de espuma para describir la escena contemporánea que muestra al ser humano en colisión con los fenómenos de la globalización. Tal como afirma Gómez Vázquez 
(2020), "En el proceso de formación de las espumas hay ganadores y perdedores. Esto explica las dinámicas de desigualdad, migración y amenazas terroristas en un espacio que el 'palacio de cristal' no logra suprimir" (117). De hecho, lo que Sloterdijk postula en este volumen es una suerte de implosión de los escenarios íntimos propios de su teoría microesférica, es decir, de aquellos concernientes al espacio intrauterino, transformados ahora en espacios interconectados expansivamente. En la idea de espuma se concentran teoría tecnológica, ingeniería y técnica climatológica:

Desde el principio tiene que quedar claro que este «leer» en las espumas no puede quedarse en mera hermenéutica, ni detenerse en el desciframiento de signos. Sólo entra en materia como teoría tecnológica de espacios humanamente habitados, simbólicamente climatizados, es decir, como instrucción científico-ingeniera y política para la construcción y mantenimiento de unidades civilizatorias, un ámbito temático que hasta ahora caía dentro de la ética y de sus ramificaciones en politología y pedagogía. (Sloterdijk, 2006d: 35)

Ahora bien, si hubiera que arriesgar un término para describir esta fase "espumosa" de la teoría de las esferas, posiblemente este sería el de una filosofía de lo "indeterminado", puesto que con la idea de espuma se hace referencia a la amplísima gama de procesos efímeros y atmosféricos, a un nuevo "complejo de reglas" donde tienen cabida lo poco llamativo y lo discreto, lo borroso y lo surreal: "Más allá del giro 'micrológico' habría que hablar de un descubrimiento de lo indeterminado, gracias al cual [...] lo no-nada, lo casi-nada, lo casual y lo informe han conseguido conectar con el ámbito de las realidades teorizables" (Sloterdijk, 2006d: 33).

Por esta vía, el enfoque esférico-ontológico de Sloterdijk intenta explicar los mecanismos de reconstrucción del espacio simbiótico prenatal en la facticidad de la existencia mortal del ser humano (Sutherland, 2017). Siguiendo al mismo Sutherland, esta maniobra "tiene la intención de subvertir la imagen filosófica de un Orbe 'bellamente redondeado' (SI, 99) (ejemplificado por el Eleático), subrayando en cambio la contingencia e inestabilidad de los recintos en el que nos envolvemos" (2017: 143). De modo que la esferología de Sloterdijk podría entenderse mejor como una fenomenología, en la que parece jugar un papel central la afectividad. Precisamente esta es la postura de Ernste: "El cuerpo vivido (Leib) es el punto de partida y a través de la encarnación constituimos el mundo" (2018: 274). Añade el profesor neerlandés: "El cuerpo vivido une el cuerpo físico (Körper) con la mente, y por tanto supera la separación del mundo exterior físico y el mundo interior mental" (Ernste, 2018: 274). La fenomenología de las esferas, puede decirse, desmantela la metafísica clásica, a manos de una recuperación de la idea de 
lo inconsistente, lo insustancial, de lo flotante como lo real (Medina Mejía, 2017). Para Sloterdijk tal idea se muestra con toda claridad, por ejemplo, en el mito del nacimiento de Afrodita, en el que parecen fundirse la potencia de engendramiento y la formación de la espuma:

El rapsoda filosofante Hesíodo, [... ] hizo algo inolvidable para la tradición occidental de la liaison entre espuma y potencia generativa por su relato del nacimiento de la espuma de la diosa Afrodita a consecuencia de una castración titánica. Gracias a esa historia lírico-macabra se ha conservado en la memoria una poesía presocrática de la espuma junto a la metafísica menospreciadora de lo efímero, que fue la que dominó después. (2006d: 36-37)

\section{Antropotécnicas, ejercicios, caparazones}

En la perspectiva de Sloterdijk, el ser humano es esencialmente técnico. Eso, podría decirse, es su naturaleza. Desde luego, tal naturaleza no se refiere a su condición orgánica, a su Physis corporal, o al menos no fundamentalmente. Se refiere, si nos fijamos en la noción de esfera y de sistemas inmunológicos, a una ontología que concibe al ser humano virtualmente como acróbata, como ejercitante. Observa Alonso Fernández (2018): "Estos ejercicios desplegados por los seres humanos crean sistemas inmunitarios — caparazones, sistemas de protección, al fin y al cabodesde los cuales el hombre puede hacer frente a su entorno" (87). En la interpretación de Castro-Gómez: "La técnica es el conjunto de acciones racionales que permiten al hombre la producción de un medio ambiente artificial, aquello que Sloterdijk denominará 'esferas”" (2012: 65). Tales transformaciones, a juicio del filósofo colombiano, debe hacerlas el ser humano necesariamente sobre sí mismo y sus propios productos: "Para poder sobrevivir como comunidad, el hombre está obligado a alcanzar un cierto grado de control sobre su propia dotación pulsional" (CastroGómez, 2012: 66). Será justo aquí donde Sloterdijk (2006b) propondrá su teoría antropotécnica y, dentro de esta, el concepto de técnicas de distanciamiento:

Lo mejor es imaginarse a las antiguas hordas como una especie de islas flotantes, que avanzan lentamente, de modo espontáneo, por los ríos de la vieja naturaleza. Se separan del medio exterior por la revolucionaria evolución de las técnicas de distanciamiento — sobre todo por la novedosa sincronía de huida y contraataquey están sujetas desde su interior por un efecto invernadero emocional, que amalga- 
ma a los miembros de la horda — a través del ritmo, la música, los rituales, el espíritu de rivalidad, los beneficios de la vigilancia y el lenguaje- en una especie de institución psicosocial total. (25)

Esta "separación del medio exterior" o "efecto invernadero emocional" presenta una evidente simetría con los planteamientos de Ortega y Gasset, el filósofo de la técnica por antonomasia del siglo xx y para quien la técnica implica la modificación de la naturaleza: "La técnica es la reforma de la naturaleza, de esa naturaleza que nos hace necesitados y menesterosos, reforma en sentido tal que las necesidades queden a ser posible anuladas por dejar de ser problema de satisfacción" (Ortega, 1983: 324). Dicha reforma de la naturaleza debe entenderse precisamente en el sentido - y aquí el parangón con Sloterdijk — de una transformación del entorno, del medio o, dicho en nomenclatura orteguiana, de la "circunstancia". Se trata, en ambos pensadores europeos, de la técnica como un conjunto de actos esenciales de la especie humana. Sin embargo, en Ortega el ser humano deberá (porque tiene la posibilidad) replegarse sobre sí mismo, ensimismarse, a fin de desarrollar justamente su proyecto técnico, que no es otro que su proyecto vital. Al respecto apunta García Madalena (2018): "En estos momentos de ensimismamiento a los que Ortega también denomina extra o sobrenaturales, ultrabiológicos, momentos de retracción en sí, es cuando el hombre realiza su segundo repertorio de actos, es decir, inventa y ejecuta” (190). Análogamente, la humanidad será para Sloterdijk una deriva de mejoramiento técnico en relación con las determinaciones del entorno, con la diferencia de que en el alemán se trata de una ontología de un espacio compartido entre lo humano y lo técnico: "Esta ontología postulará un territorio compartido, indiscernible, entre lo humano y lo técnico, que hace de esto aquello y viceversa. Las operaciones que hacen del hombre tal cosa son fundamentalmente técnicas, y la tecnicidad es lo humano por excelencia” (Ortiz Palacios y Ríos, 2018: 57). En tal sentido, y este podría ser un axioma de la teoría sloterdijkiana, el ser humano ha requerido y requiere (y, por lo mismo, ha sido capaz) de la creación de dispositivos inmunológicos que le permitan con cierto éxito hacer frente a las hostilidades de lo circundante. Escribe Alonso Fernández (2018): "hasta cierto punto podría decirse que Sloterdijk va más lejos que el propio Ortega en su caracterización de la relación entre organismo y circunstancia como antagónica y hostil, entendiendo que los organismos vivos son principalmente y antes que nada sistemas inmunitarios que anticipan y se protegen continuamente de las agresiones provenientes del entorno" (87).

Esta especie de recubrimiento o coraza de la que habla Sloterdijk, tiene su correlato en la conocida idea de Ortega de sobrenaturaleza. En efecto, para el filó- 
sofo madrileño la sobrenaturaleza equivale justamente a lo creado por la técnica, al conjunto de productos que el ser humano ha definido como necesarios para su proyecto vital, con la salvedad de que lo que se modifica finalmente es la propia naturaleza respecto de las necesidades específicamente humanas. Como acota García Madalena (2018):

La sobrenaturaleza es, en primer lugar, consecuencia de la "reacción enérgica contra la naturaleza que nos hace menesterosos" [...], esto es, la transformación impuesta por el ser humano a la naturaleza y, en segundo lugar, es una nueva naturaleza creada por el ser humano, sobrepuesta, es el mundo que inventamos y creamos con la técnica, un mundo humanizado donde transcurre la vida humana. (200)

La sobrenaturaleza orteguiana es, pues, naturaleza trascendida. Paradójicamente, en Sloterdijk el ser humano extravía su trascendencia para llegar a verse como un producto mecánico, un segundo Dios ortopédico (Fuentes, 2020).

\section{Crianza, artificialidad, astronáutica tripulada}

Pues bien, si seguimos más de cerca el proyecto de las antropotécnicas, notamos la preponderancia que Sloterdijk (2012) da a la idea de optimización de nuestro equipamiento inmunológico:

Entiendo, con esta última expresión [antropotécnicas], los procedimientos de ejercitación, físicos y mentales, con los que los hombres de las culturas más dispares han intentado optimizar su estado inmunológico frente a los vagos riesgos de la vida y las agudas certezas de la muerte. Sólo cuando estos procedimientos sean captados dentro de un amplio cuadro de los "trabajos del hombre en sí mismo" se podrán evaluar los más recientes experimentos de las técnicas genéticas, a las cuales se quiere limitar, en el debate actual, el concepto de "antropotécnica" reacuñado en 1997. (24)

Este es el mismo sentido que el filósofo dará al homo immunologicus, el cual, dotado de una armadura simbólica en su vida, se impone como el ser humano que lucha consigo mismo, preocupado por su propia forma (Sloterdijk, 2012). Dicha estructura de crianza-inmunización, siguiendo a Castro-Gómez (2012), presenta una doble dimensión: por un lado, las que cabría llamar técnicas "hacia afuera" (Fernsteuerung), que virtualmente operan como un entrenamiento selectivo de las 
mejores técnicas adaptativas que cada grupo humano selecciona en orden de su mayor efectividad. Serían, pues, técnicas preferentemente biopolíticas. Por otro lado, están las técnicas que se desarrollan más bien respecto de uno mismo ( $\mathrm{Sel}$ bststeuerung) bajo la idea de automejoramiento. Serían, en este caso, técnicas autoadaptativas o de autodomesticación.

Ahora, al insinuar que a la filosofía le cae sobre los hombros la responsabilidad de proponer algo parecido a un logos de la crianza, lo que hace Sloterdijk es reapropiarse de la moraleja filosófica del Estado platónico, que recomienda que "los que ahora son llamados reyes y gobernantes filosofen de modo genuino y adecuado" (Platón, 2000: 473d). Así, la antropotécnica real "requiere que el político sepa entretejer del modo más efectivo las propiedades de los hombres voluntariamente gobernables que resulten más favorables a los intereses públicos, de manera que bajo su mando el parque humano alcance la homeostasis óptima" (Sloterdijk, 2006c: 81). Las antropotécnicas que Sloterdijk plantea incluir como técnicas del futuro, deben romper lo más eficientemente posible con aquellas técnicas contemplativas propias del humanismo medieval y moderno. Se trata de cultivar técnicas para una "crianza de la desinhibición", o sea, técnicas que favorezcan una transformación del sujeto "para lanzarlo hacia los experimentos consigo mismo" (Castro-Gómez, 2012: 72). Evidentemente, advierte Fidalgo Benayas (2013), esta crianza preparatoria requerirá de una nueva ontología: "La respuesta de Sloterdijk a la Carta sobre el humanismo consiste en negar la negación de la animalidad del hombre y en negar la afirmación de su carácter cuasidivino. El resultado es la afirmación de la animalidad del hombre y la negación de su sacralidad" (Fidalgo Benayas, 2013: 123).

Por cierto, esta negación de la sacralidad no significa en modo alguno negar al mismo tiempo la vinculación de los sistemas inmunológicos con el mundo de la religión. Al revés, y como lo detalla Sloterdijk en Has de cambiar tu vida, las propias bases de la Edad Moderna se deben en gran medida a la recuperación de la práctica ascético-cristiana de "transformar toda su vida en un experimento, para asemejarse al Hombre-Dios” (Alonso Fernández, 2018: 89). De esta manera, la idea de técnica debe entenderse en Sloterdijk en un sentido bastante más sofisticado que el de un simple modo de transformación de las fuerzas de la naturaleza por medio de nuestro ingenio. Más bien está pensada en el sentido de mecanismos orgánicos y mentales de resolución de problemas (sociales, políticos, biomédicos, militares, económicos, etcétera) con fines inmunológicos. De ahí que Sloterdijk fustigue al proyecto humanista su incapacidad de sobreponerse a la hecatombe que supuso el fin de la metafísica en el proyecto filosófico de la modernidad, pero sobre todo su ceguera ontológica en no reconocer la importancia de la artificialidad: 
Hace tiempo que existimos en mundos vitales tecnologizados, en los que máquinas clásicas y cibernéticas son decisivas para nuestra forma de existencia. En vista de estos fenómenos evidentes resulta fácil hacer valer una interpretación de la modernidad como artificialización. La ley de lo moderno es la intervención creciente de la artificialidad en todas las dimensiones estables de la existencia. (Citado en Goycolea, 2017: 7)

La crítica de Sloterdijk precisamente le echa en cara al humanismo sobreviviente su abandono de la posibilidad de una conciencia nómade y de un cierto imperativo de "ejercitación de la vida" (Maureira, 2016). Al contrario, Sloterdijk verá en este humanismo una suerte de incubadora milenaria que ya no produce humanos, porque el escenario actual en el que se despliega el ser es el provisto por los medios de comunicación de masas, los fármacos y la biotecnología (Maureira, 2016). Vale decir, se trataría de la recuperación de una actitud xenolátrica que implique desarrollar un pensamiento ecológico en sentido amplio, que involucre tanto a vectores naturales como tecnológicos, pero a la vez centrado nuevamente en el amor por la vida desconocida, lejana y venidera en cuanto proyecto de ética acrobática con foco en las antropotécnicas (Maureira, 2016).

Sinópticamente, para Ortega "la vida humana no tiene lugar en la naturaleza, sino en esta nueva naturaleza o sobrenaturaleza" (García Madalena, 2018: 201). Esta nueva naturaleza o artificialidad parece equivaler, en la tesis de Sloterdijk, a los domos o invernaderos que el ser humano se ha creado a lo largo de toda la historia, interpretación que sólo es posible a partir de una teoría esencialmente heterodoxa de la cultura y la civilización. Tal como postula el filósofo de Karlsruhe - $-\mathrm{y}$ esta es una observación sobremanera relevante respecto de la intersección de los conceptos de técnica y esferas - la disciplina más cercana a esta teoría puede hallarse, de momento, en la astronáutica tripulada, pues en ninguna otra parte se pregunta tan radicalmente por las condiciones técnicas de la posibilidad de existencia humana en cápsulas que mantengan la vida (Sloterdijk, 2006d: 35).

\section{Conclusión}

Sloterdijk llega a desarrollar una ontología de las esferas como una virtual metafísica de la intersubjetividad espacial. Como apunta Rendón Ángel (2011), este espacio esferológico tiene una apertura inicial que siempre involucra a un segundo con el que no se presentan desniveles relacionales de ningún tipo. De este modo, siguiendo al filósofo colombiano, entre sujeto y objeto lo ontológico debe ser visto como 
un desnivel paradigmáticamente filosófico. La esfera, pues, constituye en la teoría sloterdijkiana el principio ontológico fundamental, donde el espacio, gracias a una potencia de redondeamiento advertida por Sloterdijk, "fuerza" en cualquier sitio donde haya un ente algo así como conformaciones originarias de círculos y globos. Esta especie de energeia justificaría la idea de un ser humano en una continua deriva técnico/tecnológica, de donde las burbujas, los globos y las espumas serían las conformaciones ontológicas esenciales. Por lo mismo, la construcción de esferas (el ejercicio inmunológico por antonomasia) pareciera estar, además, vinculada a la idea de conectivismo afectivo entre los distintos individuos: relaciones madre/niño, hipnotizador/sujeto, terapeuta/paciente, feto/placenta, etcétera (Duclos, 2019). Este papel de la afectividad permitiría comprender la teoría de las esferas como fenomenología (de lo dúplex, si se quiere), en el sentido de una recuperación de la idea de lo inconsistente, de lo insustancial, de lo flotante como lo real. Sería, en todo caso, una fenomenología acrobática.

Los sistemas inmunológicos en Sloterdijk, que no son otra cosa que los espacios conformados como esferas, se definen antropotécnicamente como estructuras de crianza-inmunización. Tales domos o caparazones tienen un correlato casi perfecto con la concepción de Ortega de la técnica como sobrenaturaleza. En ambos casos, tanto en Sloterdijk como en Ortega, estas corazas creadas por la técnica, en cuanto productos antropotécnicos, modifican la propia naturaleza respecto de las necesidades específicamente humanas. Así, en la intersección de las nociones de técnica y esfera (y esta es una cuestión que escapa a la pura tecnología y se encumbra precisamente al proyecto posthumanista de Sloterdijk) la disciplina más cercana a la teoría esferológica pudiera hallarse, de momento, y tal como lo subraya nuestro autor, en la astronáutica tripulada, donde hay una pregunta radical por las condiciones técnicas de la posibilidad de existencia humana en cápsulas que mantengan la vida. Desde el útero materno hasta una caminata espacial, siempre habrá un otro que nos constituya, que nos defina, que nos "acorace" decisivamente.

¿No hará falta, entonces, llegar a una interpretación de las esferas de Sloterdijk, vale decir, de su ontología, lejos de las posibilidades de la eugenesia o de la nanotecnología, de la mano del diseño y la estética, o mejor aún, aferrados a la erótica?

\section{Referencias bibliográficas}

Alonso Fernández, Marcos. (2018). "Convergencias entre la sobrenaturaleza de Ortega, la exteriorización de Stiegler y la inmunología de Sloterdijk”. Argumentos de Razón Técnica, (21), 77-91. 
Arango Flórez, Jhon. (2007). "Para una arqueología de lo íntimo". Co-herencia, 4(7), 217-222. https://publicaciones.eafit.edu.co/index.php/co-herencia/article/ view/3884.

Castro-Gómez, Santiago. (2012). "Sobre el concepto de antropotécnica en Peter Sloterdijk”. Revista de Estudios Sociales, (43), 63-73. http://dx.doi.org/10.7440/ res43.2012.06.

Duclos, Vincent. (2019). "Falling into things: Peter Sloterdijk, ontological anthropology in the monstrous". New Formations: A Journal of Culture/Theory/Politics, 95, 37-53. https://www.muse.jhu.edu/article/715818.

ERnste, Huib. (2018). "The geography of spheres: an introduction and critical assessment of Peter Sloterdijk's concept of spheres". Geographica Helvetica, 73(4), 273-284. https://doi.org/10.5194/gh-73-273-2018.

Fidalgo Benayas, Leónides. (2013). "Ontología del hombre y deconstrucción: Heidegger, Sartre / Derrida, Sloterdijk”. Eikasia, (51), 103-128.

Fuentes, Rafael. (2020, 5 de marzo). "Sloterdijk explora el octavo día de la creación". $A B C$ (en línea). ABC Cultural. Recuperado de https://www.abc.es/cultura/ cultural/abci-sloterdijk-explora-octavo-creacion-202003050100 noticia.html.

García Madalena, Alfonso. (2018). El centauro ontológico. Idea y sentido de la técnica en Ortega y Gasset (Tesis de doctorado, Universidad Nacional de Educación a Distancia). Recuperado de http://e-spacio.uned.es/fez/view/ tesisuned:Filosofia-Agarciam.

Gimeno Monfort, Xavier; López Frías, Francisco Javier. (2018). “Una crítica a la modernidad a partir de la 'inutilidad' del juego. El imperativo metanoético de Sloterdijk y la razón lúdica”. Recerca: Revista de Pensament I Anàlisi, (23), 219-238. https://doi.org/10.6035/Recerca.2018.23.10.

Gómez VÁzQuez, José Luis. (2020). “El relato histórico-filosófico de la globalización como herramienta para la reflexión literaria. De un concepto operativo a un estudio de caso". Literatura: teoría, historia, critica, 22(1), 111-136. https:// doi.org/10.15446/lthc.v22n1.82294.

GoycoleA, Mateo. (2017). "Sobre el concepto de humanismo: Heidegger y Sloterdijk”. Crítica. cl. Fundación Reflexiva. Recuperado de https://critica.cl/filosofia/sobre-el-concepto-de-humanismoheidegger-y-sloterdijk.

Huerta Rodríguez, Raúl Adrián. (2016). Esferología, politica y guerra en Peter Sloterdijk. Para una aphrología de la guerra de cuarta generación (Tesis de maestría, Universidad Iberoamericana). Recuperado de http://ri.ibero.mx/handle/ ibero/497. 
Meaney, Thomas. (2018, 26 de febrero). "A celebrity philosopher explains the populist insurgency”. The New Yorker (en línea). Recuperado de https://www.newyorker. com/magazine/2018/02/26/a-celebrity-philosopher-explains-the-populist-insurgency. Medina Mejía, Ernesto. (2017). Peter Sloterdijk: esferología y subjetividad (Tesis de maestría, Universidad Autónoma del Estado de México). Recuperado de http:// hdl.handle.net/20.500.11799/98670

Maureira, Marco. (2016). "Posthumanismo: más allá de antropotécnica y nomadismo". Cinta de Moebio, (55), 1-15. http://dx.doi.org/10.4067/S0717554 X2016000100001.

Oosterling, Henk. (2007). "Interest and excess of modern man's radical mediocrity: rescaling Sloterdijk's grandiose aesthetic strategy”. Cultural Politics, 3(3), 357-380. https://doi.org/10.2752/175174307X226898.

Ortega y Gasset, José. (1983). Obras Completas. Madrid: Alianza. 12 tomos.

Ortiz Palacios, Jimmy; Ríos, Camilo. (2018). "Hacia una actualización éticopolítica a partir de la noción de antropotécnica en Peter Sloterdijk". Cuadrante Phi, (30), 42-61. https://repository.javeriana.edu.co/handle/10554/42745.

Platón. (2000). Diálogos IV. República V. Madrid: Gredos.

Raschke, Carl. (2013). "Peter Sloterdijk as 'first philosopher' of globalization”. Journal for Cultural \& Religious Theory, 12(3). https://jcrt.org/archives/12.3/ raschke.pdf.

RENDón Ángel, Juan Edilberto. (2011). “Los duetos microsferológicos. La noción de espacio en la trilogía Esferas de Peter Sloterdijk como alternativa al individualismo". Katharsis: Revista de Ciencias Sociales, (12), 129-161.

SAfranski, Rüdiger. (2009 [2003]). "Prólogo”. En Peter Sloterdijk, Esferas I. Burbujas: microsferología. Madrid: Siruela.

Sloterdijk, Peter. (2003). Esferas I. Burbujas. Madrid: Siruela.

Sloterdijk, Peter. (2004). Esferas II. Globos. Macrosferología. Madrid: Siruela.

Sloterdijk, Peter. (2006a). "El hombre operable. Notas sobre el estado ético de la tecnología génica” [Conferencia]. Centro de Estudios Europeos (CES) de la Universidad de Harvard, Estados Unidos. Recuperado de https://www.observacionesfilosoficas.net/elhombreoperable.html.

SLOTERdijk, Peter. (2006b). En el mismo barco. Ensayo sobre la hiperpolitica. Madrid: Siruela.

Sloterdijk, Peter. (2006c). Normas para el parque humano. Madrid: Siruela.

SLOTERdijk, Peter. (2006d). Esferas III. Espumas. Esferología plural. Madrid: Siruela.

Sloterdijk, Peter. (2012). Has de cambiar tu vida. Valencia: Pre-Textos. 
Sordo, Juan; Guzmán DíAz, Ricardo. (2013). "Cultura y política en el proyecto filosófico de Peter Sloterdijk. Limitaciones y potencialidades de su esferologia”. Desacatos, (41), 139-154.

Sutherland, Thomas. (2017). "Ontological co-belonging in Peter Sloterdijk's spherological philosophy of mediation”. Paragraph, 40(2), 133-152. https:// doi.org/10.3366/para.2017.0222.

Tomaz, Tales. (2020). "Crítica da tecnologia como metafísica: reflexão sobre a narrativa pós-natureza do antropoceno". Veritas (Porto Alegre), 65(1), 1-11. https://doi.org/10.15448/1984-6746.2020.1.36679

VAN Tuinen, Sjoerd. (2007). "Critique beyond resentment: an introduction to Peter Sloterdijk's jovial modernity”. Cultural Politics, 3(3), 275-306. https:// doi.org/10.2752/175174307X226861. 